\title{
Short-wave infrared Mueller matrices and polarization parameters for in vivo skin surface reflectance
}

Lisa W. Li, Russell A. Chipman

Lisa W. Li, Russell A. Chipman, "Short-wave infrared Mueller matrices and polarization parameters for in vivo skin surface reflectance," Proc. SPIE 10655, Polarization: Measurement, Analysis, and Remote Sensing XIII, 1065503 (14 May 2018); doi: 10.1117/12.2305279

Event: SPIE Commercial + Scientific Sensing and Imaging, 2018, Orlando, Florida, United States 


\title{
Short-Wave Infrared Mueller Matrices and Polarization Parameters for in vivo Skin Surface Reflectance
}

\author{
Lisa W. Li ${ }^{\mathrm{a}}$ and Russell A. Chipman ${ }^{\mathrm{a}}$ \\ ${ }^{a}$ University of Arizona, 1630 E University Blvd, Tucson, USA
}

\begin{abstract}
Short-wave infrared light is weakly absorbed by human epidermal and dermal tissue. Investigation of polarization characteristics of human skin taken in vivo distinguishes how depolarization index and the Mueller matrix are markers that can identify skin from a rough Lambertian surface material. A custom Near-Infrared imaging Mueller Matrix Polarimeter using an expanded, coherent 1550nm laser beam source is used to analyze the skin on the back of hands as a subject.
\end{abstract}

Keywords: SWIR, polarized light, skin, mueller matrix, depolarization

\section{INTRODUCTION}

Many studies have been performed on the light interaction characteristics of skin from the ultraviolet to the visible to the near-infrared spectrum. Existing knowledge of the properties of skin is well-documented for irradiance while fewer studies ${ }^{1}$ have been carried out on properties of polarization. Attributes of reflectance, transmission, absorption, and scatter over a multi-wavelength range have been explored in previous work. ${ }^{2-4}$ Polarization characteristic are of particular interest because irradiance and polarization carry information that arise from fundamentally different sources; irradiance is a function of electron oscillation amplitude while polarization is a function of electron oscillation direction.

A shortwave infrared (SWIR) source wavelength was selected because SWIR light can penetrate further into the skin compared to light in the visible spectrum. ${ }^{5}$ Having detailed polarization characteristic information in the SWIR can enhance capabilities for medical imaging and material discrimination.

A methodology is presented for measuring and characterizing the polarization properties of in vivo skin tissue on the back of the hand by examining full Mueller matrix images measured using a Near-Infrared Imaging Mueller Matrix Polarimeter (NIRPol). By comparing the resulting skin data to measurements taken of extra fine grit sandpaper-an approximate Lambertian surface-multi-angle polarization properties are determined.

\section{BACKGROUND}

\subsection{Mueller Calculus}

Mueller Calculus is used to calculate how incident polarized states of light interact with materials to generate corresponding outgoing polarized states of light. Stokes parameters are represented by $4 \mathrm{x} 1$ column vectors whose elements correspond the polarization states that make up propagating light. Mueller matrices are a representation of how a material interacts with and changes the polarization states of incident light. Mueller matrices are used in conjunction with Stokes parameters to represent changes in incident states of light due to a material's influence.

The first element represents the irradiance of that light and is often normalized to one. The second element may have a value between -1 and +1 , where -1 represents completely horizontally polarized light while +1 represents vertically polarized light. The third element ranges from -1 to +1 , representing $-45^{\circ}$ or $135^{\circ}$ linearly polarized light where +1 represents $+45^{\circ}$ linearly polarized light. The fourth element ranges from -1 to +1 and describes the amount of left-hand circularly polarized light or right-hand circularly polarized light present.

Further author information: (Send correspondence to L.W.L)

L.W.L.: E-mail: lisali@optics.arizona.edu, Telephone: 14806035243

R.A.C.: E-mail: rchipman@email.arizona,edu, Telephone: 15206269435

Polarization: Measurement, Analysis, and Remote Sensing XIII, edited by David B. Chenault, Dennis H. Goldstein, Proc. of SPIE Vol. 10655, 1065503 - (c) 2018 SPIE · CCC code: 0277-786X/18/\$18 $\cdot$ doi: $10.1117 / 12.2305279$

Proc. of SPIE Vol. 10655 1065503-1 
Stokes parameters are multiplied with the $4 \times 4$ Mueller matrix to produce a new set of Stokes parameters to describe the resulting polarization states of the exiting light, as shown below.

$$
\left[\begin{array}{l}
S_{0}{ }^{\prime} \\
S_{1}{ }^{\prime} \\
S_{2}{ }^{\prime} \\
S_{3}{ }^{\prime}
\end{array}\right]=\left[\begin{array}{llll}
m_{00} & m_{01} & m_{02} & m_{03} \\
m_{10} & m_{11} & m_{12} & m_{13} \\
m_{20} & m_{21} & m_{22} & m_{23} \\
m_{30} & m_{31} & m_{32} & m_{33}
\end{array}\right]\left[\begin{array}{c}
S_{0} \\
S_{1} \\
S_{2} \\
S_{3}
\end{array}\right]
$$

Unpolarized light would be represented by the Stokes parameters where the first element is 1 and the rest are zero. The Mueller Matrix for air is a $4 \times 4$ identity matrix.

\subsection{Depolarization Index}

The Depolarization index is a characteristic of the Mueller matrix. It is a measure from 0 to 1 which describes how depolarizing a sample is for the reflected or transmitted light incident. A value of 0 describes an ideal depolarizer with a Mueller matrix as seen below.

$$
\left[\begin{array}{llll}
1 & 0 & 0 & 0 \\
0 & 0 & 0 & 0 \\
0 & 0 & 0 & 0 \\
0 & 0 & 0 & 0
\end{array}\right]
$$

A value of 1 describes a non-depolarizing Mueller matrix, such as the Mueller matrix for air. It is important to note that the depolarization index is closely related to but distinct from the degree of polarization, which is further explained in Ref. 6.

$$
\text { DepolarizationIndex }: D I=\frac{\sqrt{\sum_{i, j=0}^{3}\left(M_{i, j}^{2}-M_{0,0}^{2}\right)}}{\sqrt{3} M_{0,0}}
$$

Depolarization Index in this paper is calculated using Gill and Berbenau's equation ${ }^{7,8}$ given above.

\section{EXPERIMENTAL METHODS}

\subsection{Near-Infrared Imaging Mueller Matrix Polarimeter}

The Near-Infrared Mueller Matrix Imaging Polarimeter (NIRPol) was used to take the data. The NIRPol features a Photonetics Tunics-C tunable laser source operating at $6 \mathrm{~mW}$ at $1550 \mathrm{~nm}$. Light is directed from the laser through a $400 \mu \mathrm{m}$ diameter multi-mode optical fiber with 0.39NA. The source is then expanded to a collimated beam of 1 inch diameter. Coherence is scrambled using a vibrating motor attached to the optical fiber.

Next, the polarization state generator (PSG) made of a linear polarizer and $\lambda / 3$ retarder create the incident polarization state that will interact with a given sample. A polarization state analyzer (PSA) made up of a $\lambda / 3$ retarder followed by a linear polarizer then picks out specific outgoing polarization states to then be imaged by the NIRPols camera. This system uses a 100mm F/2.25 SWIR Edmund optics camera lens imaging onto an InGaAs focal plane array $(320 \times 256 \mathrm{px})$ that features a response from $900 \mathrm{~nm}$ to $1700 \mathrm{~nm}$.

To capture multiple angles of incidence, the NIRPol is fitted with goniometric arms capable of capturing irradiance over a dynamic range of $0^{\circ}$ to $90^{\circ}$ angle of incidence and scatter. The minimum tolerable angle of separation between the analyzer and generator arms is $20^{\circ}$ of angular separation; mounting of the optics in the system defines this minimum.

Retroreflection measurements are possible by use of a non-polarizing cube beamsplitter, but require additional steps of computationally removing the effects of the cube to isolate a Mueller matrix which describes only the effects of skin. However, retroreflection measurements were omitted from this study after considering the fringing in the measurements presented by the use of a coherent laser source for illumination. 


\subsubsection{Measuring the Mueller Matrix}

To extract the Mueller Matrix representation of a sample, the polarimeter must measure the outgoing irradiance over a variety of polarization states coupled with a variety of input polarization states. The PSA and PSG use rotating retarders that both step in 64 equal steps over $360^{\circ}$ and $1800360^{\circ}$ to measure the irradiance values that exit from each paired PSG and PSA position. Calculation of the Mueller matrix from these irradiance measurements follows the methods outlined in chapter 22 of Ref. 9.

\subsubsection{Near-Infrared Polarimeter Accuracy}

Partial calibration and accuracy for the NIRPol is determined through the analysis of an air measurement. The expected theoretical outcome air measurement is a $4 \mathrm{x} 4$ identity matrix; ideally no effect is seen on diattenuation, polarizance, retardance, or depolarization. Acceptable tolerances for the instrument were 0.01 for diattenuation magnitude, 0.01 for polarizance magnitude, $0.1^{\circ}$ for retardance, and 0.01 for depolarization when all values are expected to be zero. Test air measurement data is taken at the start of each measurement session to ensure the calibration file loaded by the instrument is valid.

\subsubsection{Polarimeter Irradiance}

The irradiance from the 1550nm laser first transmits through 6 meters of multimode optical fiber. Next, the irradiance diminishes as the expanded beam passes through the linear polarizer that forms part of the PSG. Approximately $.5 \mathrm{~mW} / \mathrm{cm}^{2}$ or $1.5 \mathrm{~J} / \mathrm{cm}^{2} / \mathrm{h}$ of $1550 \mathrm{~nm}$ radiation is incident upon the measured surface of the back of the hand. Exposure to IR-B radiation in the summertime is $75 \mathrm{~J} / \mathrm{cm}^{2} / \mathrm{h}$, and $72 \%$ of the IR-B light that is incident upon the surface of human skin is absorbed in the epidermal layer. ${ }^{10}$ Exposure presented to a participant's hand is far lower than what is experienced when stepping outdoors during summertime.

\section{2 in vivo Skin Measurements}

Previous studies ${ }^{1,5}$ have shown the magnitude of optical activity present in skin tissues to be in direct relation to the density and orientation of the collagen fibers in skin. ${ }^{4}$ Additionally, skin of color has been measured as having differing sizes of collagen fiber bundles and a thicker, more compact dermis layer in comparison to Caucasian skin. ${ }^{11}$

Skin on the back of the hand was used as the subject of the measurement for ease of control of movement. Collagen in this region is classified as type I and is prone to displaying positive birefringence. ${ }^{1}$ The dermis layer on the back of the hand is thinner than in comparison to the dermis layer of skin on most of the rest of the body. Age has also been shown to be a significant factor for the collagen content in a human skin, with the dermal layer thinning with age. The expected penetration depth of the laser source is a few hundred microns, with the majority of the reflectance being from the upper $100 \mu \mathrm{m}$ of tissue. ${ }^{12}$ The outermost layer of skin, the epidermis, is on average $100 \mu \mathrm{m}$ thick.

Non-invasice skin measurements were taken of one volunteer, a female age 20 of mixed East Asian/Caucasian descent. All reported values are computed from an aggregate of data collected from this singular volunteer. The volunteer sat with their hand grasping a handle for stability. To further ensure stability, supports at the elbow and upper arm were available to rest on. The 1-inch diameter regions of interest included the flat expanse of skin near the base of the thumb, the flat portion of the back of the hand adjacent to the wrist, and the center of the back of the hand just below the middle finger knuckle.

The back of the hand is positioned in the focus of the camera at the coincident optical and mechanical axes of the rotating arm motors. Angles of incidence at 10,20,30, and 40 degrees were taken for each skin sample. All images were taken with the camera positioned at the specular angle. Each sampled region is 100x100 pixels in size covering a $2.5 \times 2.5 \mathrm{~cm}$ region, as seen in Figure 1.

\subsection{Real Lambertian Surface Measurements}

Extra fine sandpaper samples were used as control Lambertian materials and measured following the same procedure used for the skin measurements. Sandpaper grits of P400, P800, and P1000 were used as control Lambertian surfaces against which the skin measurements were compared. These grits have average nominal particle diameters of 35, 21.8, and $18.3 \mathrm{~m}$ respectively. Example Mueller matrix images for sandpaper and skin measurements at $\pm 10^{\circ}$ angle of incidence (AoI) and angle of scatter (AoS) is shown in Figure 2. 


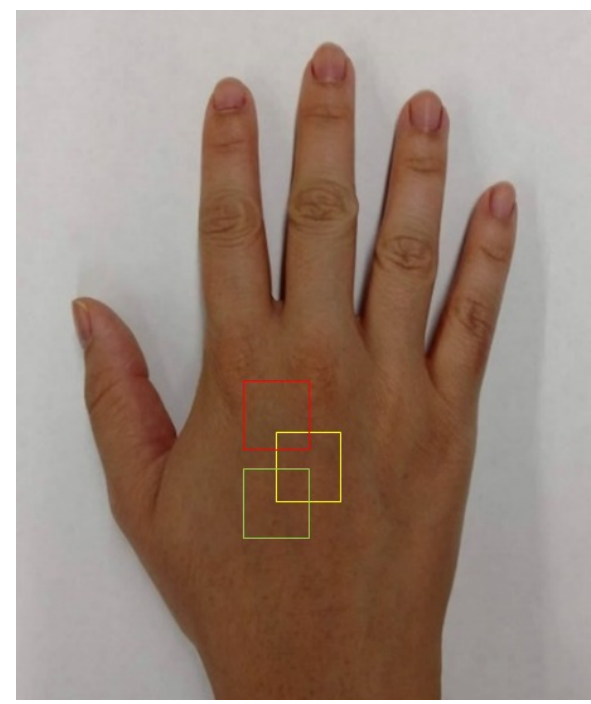

Figure 1. Photo of the back of the hand with outlined example regions of skin measured.

Table 1. Number of measurements per angle of incidence/specular angle of scatter pair for sandpaper and skin.

\begin{tabular}{|c|c|c|}
\hline Angle & Sandpaper Samples & Skin Samples \\
\hline $10^{\circ}$ & 5 & 5 \\
\hline $20^{\circ}$ & 5 & 5 \\
\hline $30^{\circ}$ & 5 & 5 \\
\hline $40^{\circ}$ & 5 & 6 \\
\hline
\end{tabular}

\section{RESULTS}

All calculations were performed using the polarization ray tracing software Polaris-M from Airy Optics ${ }^{13}$ running in Mathematica 11.2.

In order to neutralize shadowed regions adjacent to flatter plane areas of interest, a mask created from the identity matrix multiplied by a factor of $10^{-8}$ was applied to those corresponding Mueller matrix image pixels.

\subsection{Mueller Matrices}

Table 2 and Table 3 give average element values for a normalized Mueller Matrix of skin and sandpaper respectively. For a normalized Mueller matrix, the matrix is divided by the m00 element. For all other elements, the range of possible values is from -1 to 1 . The Mueller matrix values given in Table 2 and Table 3 were generated from normalized individual Mueller matrices within the whole 100x100 pixel Mueller matrix image. 

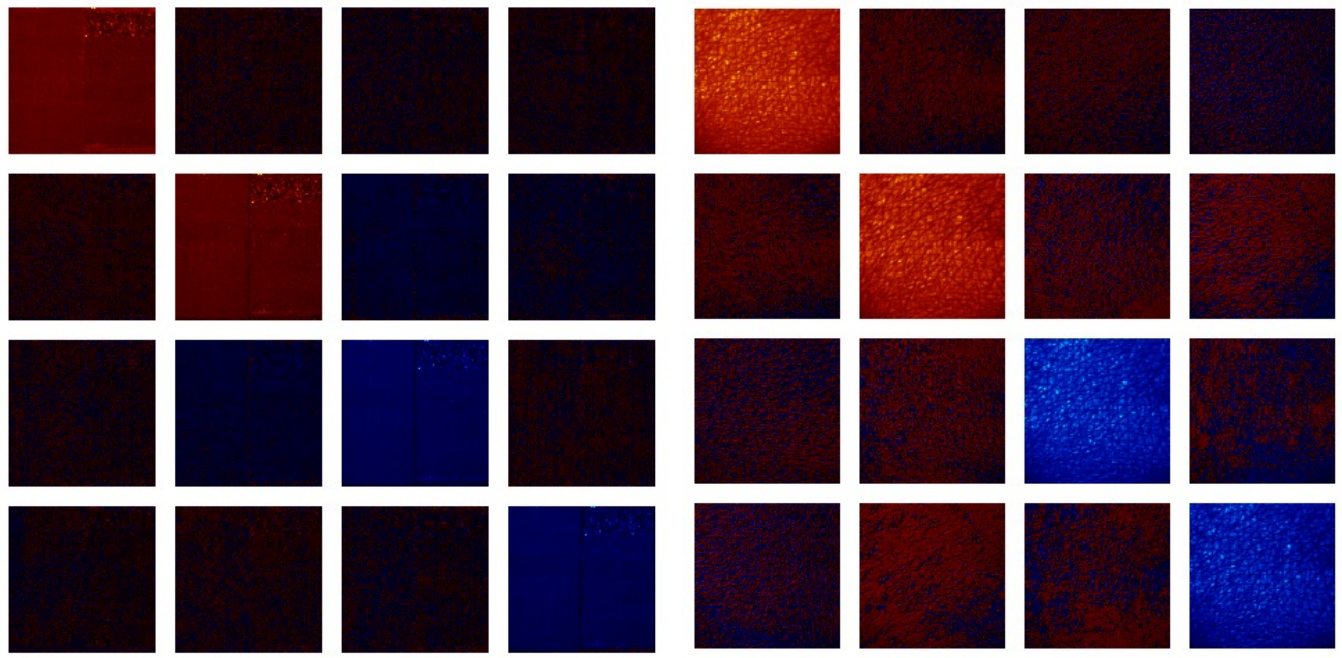

Sandpaper Mueller Matrix Image

Skin Mueller Matrix Image
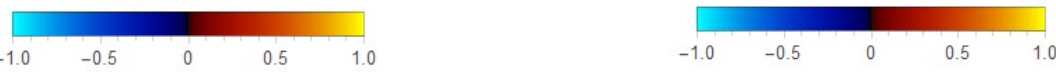

Figure 2. Mueller matrix image of assorted grit sandpapers (left) and a featureless patch of skin (right) measured at $\pm 10^{\circ}$ angle of incidence/angle of scatter.

Table 2. Average normalized Mueller matrix element values for skin

\begin{tabular}{|c|c|c|c|c|}
\hline$\theta$ & $\mathrm{m} 00$ & $\mathrm{~m} 01$ & $\mathrm{~m} 02$ & $\mathrm{~m} 03$ \\
\hline $10^{\circ}$ & 0.365067 & 0.000857941 & 0.00810332 & -0.0000145923 \\
\hline $20^{\circ}$ & 0.4258 & 0.0320875 & 0.0138679 & -0.00518358 \\
\hline $30^{\circ}$ & 0.11516 & 0.034897 & 0.00045479 & 0.00182766 \\
\hline $40^{\circ}$ & 0.0313667 & 0.00603684 & 0.00562446 & -0.000884251 \\
\hline \hline$\theta$ & $\mathrm{m} 10$ & $\mathrm{~m} 11$ & $\mathrm{~m} 12$ & $\mathrm{~m} 13$ \\
\hline $10^{\circ}$ & 0.0639612 & 0.123499 & 0.103689 & 0.0107821 \\
\hline $20^{\circ}$ & 0.122951 & 0.210268 & 0.0967096 & 0.00254768 \\
\hline $30^{\circ}$ & 0.0378906 & 0.103202 & 0.00210791 & 0.00619503 \\
\hline $40^{\circ}$ & 0.0217184 & 0.00992276 & 0.00871402 & -0.000136832 \\
\hline \hline$\theta$ & $\mathrm{m} 20$ & $\mathrm{~m} 21$ & $\mathrm{~m} 22$ & $\mathrm{~m} 23$ \\
\hline $10^{\circ}$ & -0.0142521 & 0.109191 & -0.13136 & -0.00881218 \\
\hline $20^{\circ}$ & -0.00656556 & 0.117088 & -0.199549 & -0.0050798 \\
\hline $30^{\circ}$ & 0.00360586 & 0.00419034 & -0.098068 & -0.00636208 \\
\hline $40^{\circ}$ & 0.000150883 & 0.00618623 & -0.00679704 & $-3.00366 *$ \\
\hline \hline$\theta$ & $\mathrm{m} 30$ & $\mathrm{~m} 31$ & $\mathrm{~m} 32$ & $\mathrm{~m} 33$ \\
\hline $10^{\circ}$ & -0.0000929671 & 0.0180093 & 0.016706 & -0.10618 \\
\hline $20^{\circ}$ & -0.00980252 & 0.00279597 & 0.00921885 & -0.180976 \\
\hline $30^{\circ}$ & 0.00182821 & 0.00921902 & 0.00892717 & -0.0838339 \\
\hline $40^{\circ}$ & 0.000495266 & -0.00071653 & 0.000886903 & -0.00663991 \\
\hline
\end{tabular}


Table 3. Average normalized Mueller matrix element values for sandpaper

\begin{tabular}{|c|c|c|c|c|}
\hline$\theta$ & $\mathrm{m} 00$ & $\mathrm{~m} 01$ & $\mathrm{~m} 02$ & $\mathrm{~m} 03$ \\
\hline $10^{\circ}$ & 0.99048 & 0.0131226 & 0.00734507 & -0.00714125 \\
\hline $20^{\circ}$ & 0.9876 & 0.108772 & 0.00450337 & -0.00913106 \\
\hline $30^{\circ}$ & 0.9686 & 0.262558 & -0.00322619 & -0.0125949 \\
\hline $40^{\circ}$ & 0.86364 & 0.406304 & -0.005914 & -0.00458063 \\
\hline \hline$\theta$ & $\mathrm{m} 10$ & $\mathrm{~m} 11$ & $\mathrm{~m} 12$ & $\mathrm{~m} 13$ \\
\hline $10^{\circ}$ & 0.023032 & 0.806995 & 0.0138748 & -0.00846105 \\
\hline $20^{\circ}$ & 0.124041 & 0.868513 & 0.0231657 & -0.0105822 \\
\hline $30^{\circ}$ & 0.265755 & 0.856727 & 0.0285336 & -0.0217629 \\
\hline $40^{\circ}$ & 0.439208 & 0.721598 & 0.0325913 & -0.021413 \\
\hline \hline$\theta$ & $\mathrm{m} 20$ & $\mathrm{~m} 21$ & $\mathrm{~m} 22$ & $\mathrm{~m} 23$ \\
\hline $10^{\circ}$ & 0.0246516 & 0.0288955 & -0.811171 & -0.0064651 \\
\hline $20^{\circ}$ & 0.0322602 & 0.033757 & -0.862899 & -0.0132512 \\
\hline $30^{\circ}$ & 0.0467994 & 0.0352916 & -0.798052 & -0.0272764 \\
\hline $40^{\circ}$ & 0.0441475 & 0.039354 & -0.564441 & -0.0376811 \\
\hline \hline$\theta$ & $\mathrm{m} 30$ & $\mathrm{~m} 31$ & $\mathrm{~m} 32$ & $\mathrm{~m} 33$ \\
\hline $10^{\circ}$ & 0.000598898 & 0.0183996 & 0.0115603 & -0.709525 \\
\hline $20^{\circ}$ & 0.000142012 & 0.0186271 & 0.0162177 & -0.770613 \\
\hline $30^{\circ}$ & 0.00580812 & 0.0166007 & 0.0256936 & -0.725311 \\
\hline $40^{\circ}$ & 0.0015762 & 0.0117454 & 0.0331596 & -0.53169 \\
\hline
\end{tabular}
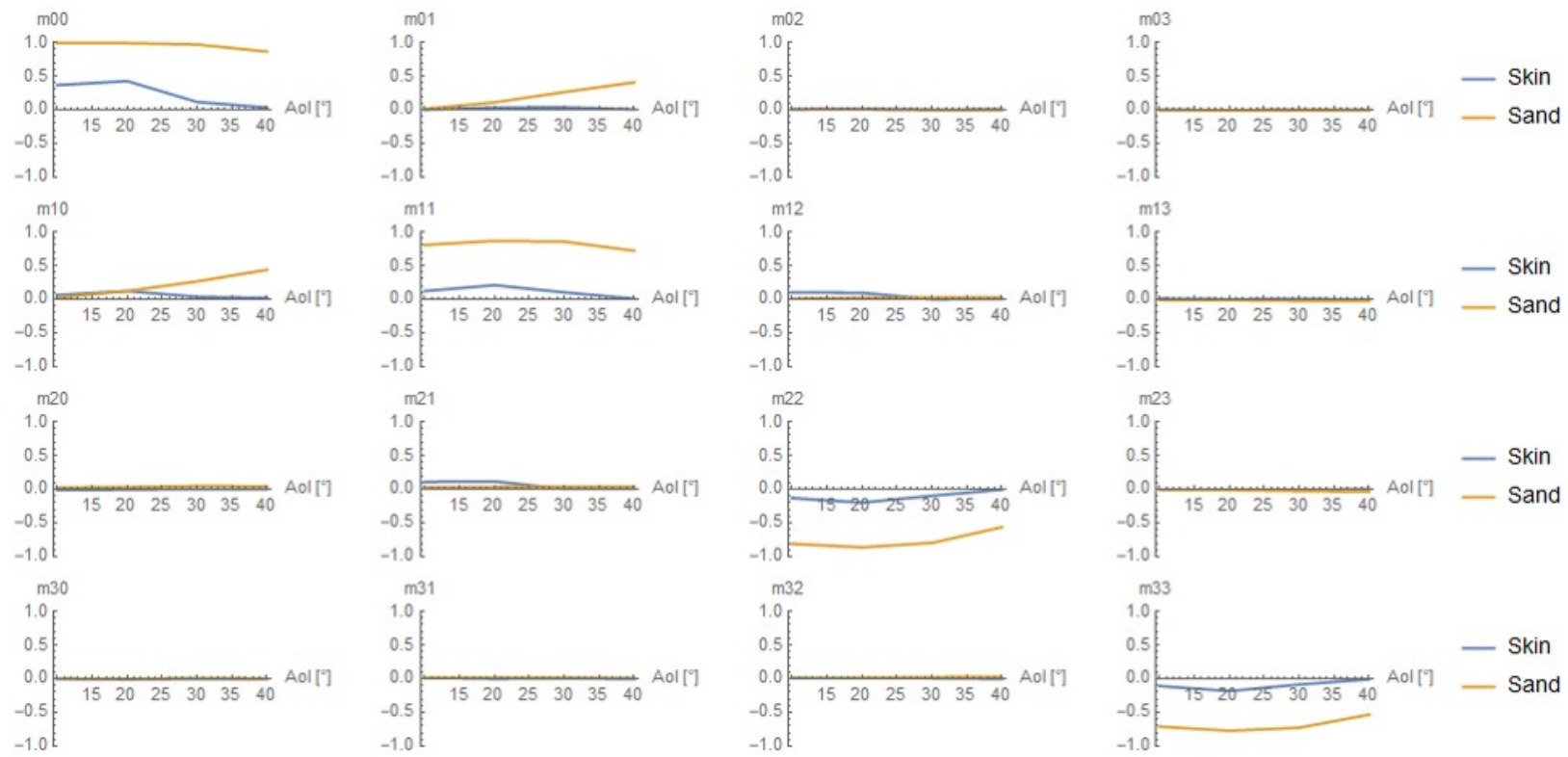

Figure 3. Average Mueller matrix element values for sandpaper and skin over $10-40^{\circ}$ angle of incidence with a specular angle of scatter.

\subsection{Depolarization Index}

Table 4 shows the depolariation index values from $10^{\circ}$ to $40^{\circ}$. Figure 4 gives a visual comparison of the skin versus sandpaper data at each angle. 
Table 4. Depolarization Index for Skin and Sandpaper

\begin{tabular}{|c|c|c|}
\hline$\theta$ & Skin & Sandpaper \\
\hline $10^{\circ}$ & 0.423218 & 0.785443 \\
\hline $20^{\circ}$ & 0.536364 & 0.85193 \\
\hline $30^{\circ}$ & 0.871753 & 0.852003 \\
\hline $40^{\circ}$ & 0.535085 & 0.815391 \\
\hline
\end{tabular}

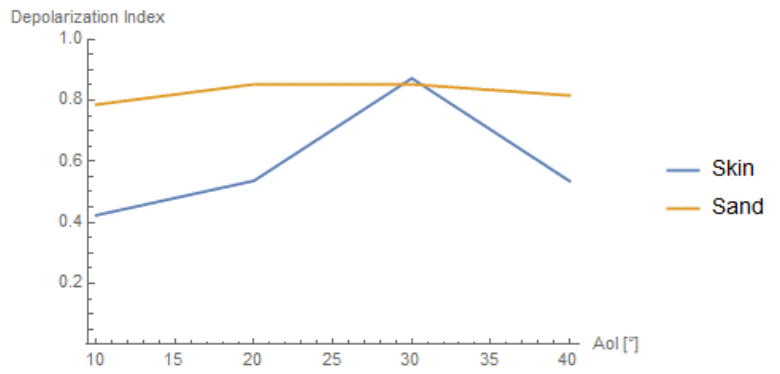

Figure 4. Average depolarization index values for sandpaper and skin over $10-40^{\circ}$ angle of incidence with a specular angle of scatter.

\section{CONCLUSION}

The Near-Infrared Polarimeter measures skin polarization in a non-invasive manner. When compared against sandpaper as a generalized rough Lambertian source, deviation in the skin data is most clearly seen in the depolarization index at $30^{\circ}$. Here, skin shows anomalous behavior in comparison to the control data. This anomaly at $30^{\circ}$ could be attributed to the effects of collagen present in the dermal layer. ${ }^{4}$ Further study would need to be conducted in order to assess the source of the depolarizing effects.

Skin layer thicknesses vary across both ethnic skin of color and regions of skin. Skin of color commonly has a thicker epidermal layer consisting of more collagen; an attempt to mitigate this variance was made by measuring skin on the back of the hand which has thinner layers. However, the thinner layers makes it compositionally different from the majority of skin on the rest of the body. Future work may want to sample from a wider range of skin types from different regions on the body to compare retardance effects in the near-infrared for many different ethnicities and mixed-race individuals. The $30^{\circ}$ parameter differences could be attributed to the epidermis and the cell content and orientation within that layer, but in order to determine the precise thickness of the layers of skin of any volunteer, a biopsy of the region of interest would be required.

\section{ACKNOWLEDGMENTS}

The authors thank undergraduate research assistants Adriana Stohn, Erica Mohr, Quinn Jarecki, Momoka Sugimura, and Cedar Andre of the University of Arizona Polarization Lab for their assistance with gathering data.

\section{REFERENCES}

[1] Tuchin, V. V., "Polarized light interaction with tissues," Journal of Biomedical Optics 21, $21-21-37$ (2016).

[2] Du, Y., Hu, X. H., Cariveau, M., Ma, X., Kalmus, G. W., and Lu, J. Q., "Optical properties of porcine skin dermis between $900 \mathrm{~nm}$ and $1500 \mathrm{~nm}, "$ Physics in Medicine $\mathscr{E}$ Biology 46(1), 167 (2001).

[3] Ding, H., Lu, J. Q., Jacobs, K. M., and Hu, X.-H., "Determination of refractive indices of porcine skin tissues and intralipid at eight wavelengths between 325 and 1557nm," J. Opt. Soc. Am. A 22, 1151-1157 (Jun 2005). 
[4] Jacques, S. L., "Corrigendum: Optical properties of biological tissues: a review," Physics in Medicine 8 Biology 58(14), 5007 (2013).

[5] Mustafa, F. H. and Jaafar, M. S., "Comparison of wavelength-dependent penetration depths of lasers in different types of skin in photodynamic therapy," Indian Journal of Physics 87, 203-209 (Mar 2013).

[6] Chipman, R. A., "Depolarization index and the average degree of polarization," Appl. Opt. 44, 2490-2495 (May 2005).

[7] Gil, J. J. and Bernabeu, E., "A depolarization criterion in mueller matrices," Optica Acta: International Journal of Optics 32(3), 259-261 (1985).

[8] Gil, J. J. and Bernabeu, E., "Depolarization and polarization indices of an optical system," Optica Acta: International Journal of Optics 33(2), 185-189 (1986).

[9] Bass, M., DeCusatis, C., Enoch, J., Lakshminarayanan, V., Li, G., Macdonald, C., Mahajan, V., and Van Stryland, E., [Handbook of Optics, Third Edition Volume II: Design, Fabrication and Testing, Sources and Detectors, Radiometry and Photometry], McGraw-Hill, Inc., New York, NY, USA, 3 ed. (2010).

[10] Schieke, S., Schroeder, P., and Krutmann, J., "Cutaneous effects of infrared radiation: from clinical observations to molecular response mechanisms.," Photodermatol Photoimmunol Photomed 19, 228-34 (Oct 2003).

[11] Vashi, N. A., Maymone, M. B. D. C., and Kundu, R. V., "Aging differences in ethnic skin," Clinical and Aesthetic Dermatology 9(1), 31-38 (2016).

[12] Ding, H., Lu, J. Q., Wooden, W. A., Kragel, P. J., and Hu, X.-H., "Refractive indices of human skin tissues at eight wavelengths and estimated dispersion relations between 300 and $1600 \mathrm{~nm}$," Physics in Medicine $\mathcal{E}^{\circ}$ Biology 51(6), 1479 (2006).

[13] Russell A. Chipman, W. S. T. L., "The polaris-m ray tracing program," (2015). 